\title{
HST/FGS Photometry of Planetary Transits of HD 209458
}

\author{
A.B. Schultz, W. Kinzel, M. Kochte, I.J.E. Jordan, F. Hamilton \\ (CSC/STScI), G. Henry (TSU), S. Vogt (UCSC), F. Bruhweiler \\ (IACS/CUA), A. Storrs (Towson University), H.M. Hart (CSC/JHU), D. \\ Bennum, J. Rassuchine, M. Rodrigue (UNR), D.P. Hamilton (UMD), W.F. \\ Welsh (SDSU), and D.C. Taylor (STScI)
}

\begin{abstract}
We present the data, and modeling and analysis results from the photometric monitoring of five planetary transits of HD 209458 using the Fine Guidance Sensors (FGS) onboard the Hubble Space Telescope (HST). We have now included the output from all four FGS photometers in our data reduction and analysis increasing our $\mathrm{S} / \mathrm{N}$ over our previous results. We have modeled the transits as an opaque spherical planet in a circular orbit about a limb darkened spherical star and simultaneously fit the model to the FGS data and published STIS transit data (Brown et al., 2001). The measured light curves show small features, a fraction of the transit depth. Some of these faint bumps and ripples appear to be real. We present an analysis of the FGS transit light curves, showing the results of the model fitting, a search for a possible planetary satellite, and an indication of which small features in the light curve we feel are not instrumental noise.
\end{abstract}

\section{INTRODUCTION}

Radial velocity observations of nearby solar-like stars have been used to detect 117 Jupiter-sized planets orbiting 102 stars. Of these Doppler-detected extra-solar giant planets (EGPs), sixteen could be classified as "51 Peg-like" or "roasters." Roaster planets are characterized by small orbital distances ( $\sim 0.1 \mathrm{AU})$, high effective temperatures (900 K $\leq \mathrm{T} \leq 1500 \mathrm{~K}$ ), and expanded atmospheres. The Doppler technique used to detect extrasolar planets provides only the minimum mass $\left(\mathrm{M}_{p} \sin (i)\right)$. The minimum masses for these planets are between $\sim 0.44$ and $\sim 1.3 \mathrm{M}_{J u p}$. Knowledge of the stellar mass combined with modeling the precise photometric transit measurements provides estimates of the basic system parameters such as the orbital inclination and planetary radius. Determining the orbital inclination removes the $\sin (i)$ dependency in the planetary mass estimate. To date, only two planets (OGLE-TR-56b, HD 209458b) have been observed to transit their host stars.

Henry et al. (1999) reported the first detection of a transit for HD 209458b. The minimum mass for HD 209458b as determined by the Doppler technique is $\mathrm{M}_{p} \sin (i)$ $\sim 0.62 \mathrm{M}_{\text {Jup }}$ (Henry et al. 2000, Charbonneau et al. 2000). The transit fixes the orbital inclination at $i=86.6^{\circ} \pm 0.14$ (Brown et al. 2001), which leaves HD 209458b with a true mass of $\sim 0.63 \mathrm{M}_{\text {Jup }}$ : quite comfortably a planet-sized body. 


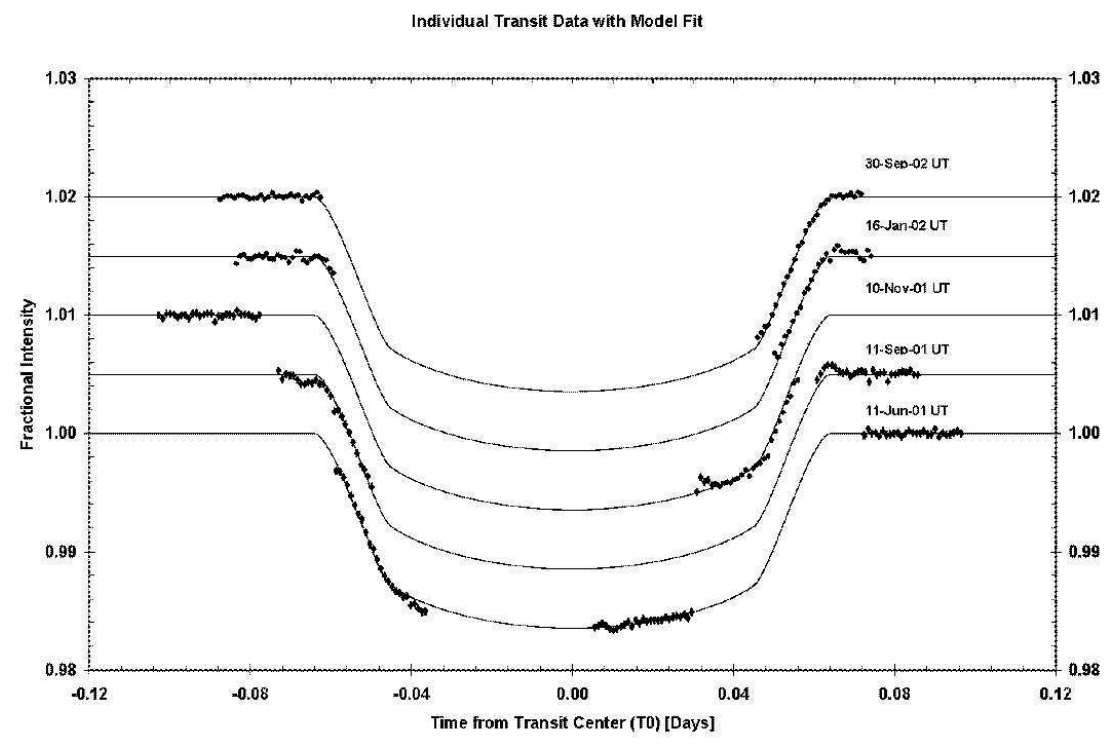

FIGURE 1. Five planetary transits of HD 209458. The in-transit data were normalized to the out-oftransit data. An arbitary off-set was added to individual transits for display purposes.

\section{OBSERVATIONS AND DATA REDUCTION}

HD 209458b is the only transiting extra-solar planet that is observable with the FGS. Five transits were observed: June 11, 2001, September 11, 2001, November 10, 2001, January 16, 2002, and September 30, 2002. FGS1r with its F550W filter $(\lambda=5500 \AA$, $\delta \lambda=5100-5875 \AA$ ) was used to obtain the data. An FGS contains four standard photomultiplier tubes (PMTs) used as high-speed photometers operating in a counting mode at $40 \mathrm{~Hz}(0.025 \mathrm{sec})($ Nelan and Makidon 2002). The expectation for each observation was to begin before the predicted time of ingress (and egress), and to capture the ingress and egress turnover points. Total integration times would depend on the available ontarget time per HST orbit minus the time for FGS setup and acquisition of guide stars. Each PMT yielded $\sim 6,500$ counts per $0.025 \mathrm{sec}$ sample per PMT ( $\mathrm{S} / \mathrm{N} \sim 80$ ).

This was the first use of the FGS to observe a bright source (HD 209458, mV=7.64) for more than a few minutes. The uncalibrated data display a time dependency in the FGS response. In addition, HST orbit phase dependent lower level variations were captured in the data, probably caused by HST breathing. The FGS dead time correction was applied to the data, drop outs were removed, and the data were placed into one second bins. To remove the time variable telescope/PMT response, a 5th order Chebyshev polynomial was fit to the out-of-transit data for each transit, and each PMT. The resulting curve was divided into each visit's data. For each time point, the 4 channel data were combined using a weighted average, where the weight is the square of the RMS from the out-oftransit data Chebyshev fit. Finally, the data were placed into 80 second bins prior to model fitting to match the STIS sampling time. The normalized data for the 5 transits are presented in Figure 1.

The June 2001 observation consisted of three contiguous HST orbits, to verify the 
observing strategy and to capture the mid-transit point for fitting purposes. The September 2001, November 2001, January 2002, and September 2002 observations consisted of two non-contiguous HST orbits, each with one non-HD 209458 orbit between the two orbits on HD 209458. The September 2001 and January 2002 observations caught the ingress and egress turn over points.

\section{LIGHTCURVE MODELING}

We have simultaneously fit the FGS and STIS transit data (Brown et al. 2001), via $\chi^{2}$ minimization, to a model consisting of an opaque spherical planet in circular orbit, transiting a limb darkened star. There are seven free parameters: time of transit center $\mathrm{T}_{0}$, stellar radius $\mathrm{R}_{*}$, planetary radius $\mathrm{R}_{p}$, orbital inclination $(i)$, period $(\mathrm{P})$, and the stellar limb darkening parameters $\mathrm{u}_{1}$ and $\mathrm{u}_{2}$. The star was assumed to have a mass of $1.1 \pm 0.1$ $\mathbf{M}_{\odot}$ (Mazeh et al. 2000) and quadratic limb darkening of the form:

$$
I(\mu) / I(1)=1-u_{1}(1-\mu)-u_{2}(1-\mu)^{2}
$$

where $\mu$ is the cosine of the angle between the line of sight and the stellar surface normal. The parameters were fit to the time of transit center $\mathrm{T}_{0}$ on November 10, 2001.

The uncertainty in each model parameter value was estimated using the technique given in Press et al. (2002). Using the parameter values obtained from the fit to the FGS and STIS data, 100 simulated data sets were generated. Each simulated datum was varied from the predicted normalized flux value by the addition of random noise whose magnitude was based upon the RMS of the fit to the data. Each simulated data set was then fit using the same model and free parameters as before. In addition, for each fit, the assumed mass of the star was varied based upon its uncertainty $\left( \pm 0.1 M_{\odot}\right.$, Mazeh et al. 2000). The standard deviation of each parameter obtained from the fits to the 100 simulated data sets is used as the uncertainty for that parameter. The results of this fit are listed in Table 1. The limb darkening coefficients for the STIS and FGS data are different due to the different bandpasses used.

\section{ANALYSIS AND CONCLUSIONS}

The fit results are consistent with previous observations (Henry et al. 2000, Charbonneau et al. 2000, Castellano et al. 2000, Robichon \& Arenou 2000, Brown et al. 2001). The inclusion of the data from all four FGS PMTs allowed for a more consistent data set which is better fit by the model as compared to previously reported results (Schultz et al. 2002). In addition, they decreased the parameter uncertainties in some cases by a factor of 10.

The STIS data for each transit was obtained using 5 contiguous HST orbits. The continuous pointing allowed HST to equilibrate. All but the first transit observed with the FGS only used 2 non-contiguous HST orbits. For each of these transits, HST was pointed at targets unrelated to this program during the mid-transit times. The changing HST attitude did not allow the temperatures of the telescope to stabilize and made it 
TABLE 1. Fit to the HST FGS \& STIS Observations of HD 209458.

\begin{tabular}{crr}
\hline & \multicolumn{2}{c}{ Data Fit } \\
& value & uncertainty \\
\hline $\mathrm{T}_{0}$ & 2452223.895819 & 0.000031 \\
\hline $\mathrm{R}_{*}\left(\mathrm{R}_{\odot}\right)$ & 1.154 & 0.036 \\
\hline $\mathrm{R}_{p}\left(\mathrm{R}_{J}\right)$ & 1.367 & 0.043 \\
\hline inclination $i$ & $86 .{ }^{\circ} 525$ & $0 .{ }^{\circ} 054$ \\
\hline Period (days) & 3.52474408 & 0.00000029 \\
\hline $\mathrm{u}_{1}($ FGS $)$ & 0.549 & 0.054 \\
\hline $\mathrm{u}_{2}($ FGS $)$ & -0.043 & 0.081 \\
\hline $\mathrm{u}_{1}(\mathrm{STIS})$ & 0.347 & 0.040 \\
\hline $\mathrm{u}_{2}(\mathrm{STIS})$ & 0.228 & 0.066 \\
\hline$\chi^{2}$ & 2.7 \\
\hline
\end{tabular}

difficult to calibrate the PMT gain changes. Thus, some of the individual features in the FGS data at or below about $0.1 \%$ may be calibration artifacts.

To date, none of the analysis has indicated the presence of a moon. A moon of 2-Earth radii in size with a 1.5 day period, resulting in a $\sim 0.1 \%$ dip in light intensity, would have been easily detected in the data. Tides would cause a moon of this size to decay into the planet in a time scale of order $10^{5}$ years. One of the remaining tasks is to determine the upper limits for the radius and mass of an undetected hypothetical moon.

\section{ACKNOWLEDGMENTS}

This work was supported by NASA through HST General Observer grant GO-09171.01A from the Space Telescope Science Institute (STScI).

\section{REFERENCES}

1. Brown, T. M., et al. 2001, ApJ, 552, 699.

2. Castellano, T., et al. 2000, ApJ, 532, L51.

3. Charbonneau, D., Brown, T.M., Latham, D.W., and Major, M. 2000, ApJ, 529, L45.

4. Henry, G., Marcy, G., Butler, R. P., and Vogt, S. S. 1999, IAU Circ. 7307.

5. Henry, G., Marcy, G., Butler, R. P., \& Vogt, S. S. 2000, ApJ, 529, L41.

6. Mazeh, T., et al. 2000, ApJ, 532, L55.

7. Nelan, E., and Makidon, R., HST Data Handbookfor FGS, 4.0, (2002), URL http : / / www . st sci. edu/instruments/fgs.

8. Press, W., et al. 2002, Numerical Recipes in $C++$, Section 15.6, Cambridge University Press.

9. Robichon, N. and Arenou, F. 2000, A\&A, 355, 295.

10. Schultz, A.B. et al. 2002, in Scientific Frontiers in Research on Extrasolar Planets, ASP Conf. Ser. 294, 479. 